\title{
Polynomials of small degree evaluated on matrices
}

\author{
Zachary Mesyan
}

November 1, 2018

\begin{abstract}
A celebrated theorem of Shoda states that over any field $K$ (of characteristic 0), every matrix with trace 0 can be expressed as a commutator $A B-B A$, or, equivalently, that the set of values of the polynomial $f(x, y)=x y-y x$ on $\mathbb{M}_{n}(K)$ contains all matrices with trace 0 . We generalize Shoda's theorem by showing that every nonzero multilinear polynomial of degree at most 3 , with coefficients in $K$, has this property. We further conjecture that this holds for every nonzero multilinear polynomial with coefficients in $K$ of degree $m$, provided that $m-1 \leq n$.
\end{abstract}

Keywords: multilinear polynomial, matrix, trace

2010 MSC numbers: 15A54, 16S36, 16S50

\section{Introduction}

We begin by recalling a theorem, which was originally proved for fields of characteristic 0 by Shoda [12] and later extended to all fields by Albert and Muckenhoupt [1].

Theorem 1 (Shoda/Albert/Muckenhoupt). Let $K$ be any field, $n \geq 2$ an integer, and $M \in \mathbb{M}_{n}(K)$. If $M$ has trace 0 , then $M=A B-B A$ for some $A, B \in \mathbb{M}_{n}(K)$.

For any $A, B \in \mathbb{M}_{n}(K)$ the traces of $A B$ and $B A$ are equal, and therefore any matrix that can be expressed as a commutator $(A B-B A)$ must have trace 0. (This observation is perhaps what prompted Shoda to prove the above result.) Thus, using $\left[\mathbb{M}_{n}(K), \mathbb{M}_{n}(K)\right]$ to denote the $K$-subspace of $\mathbb{M}_{n}(K)$ consisting of the matrices of trace 0 , and $f\left(\mathbb{M}_{n}(K)\right)$ to denote the set of values of a polynomial $f$ on $\mathbb{M}_{n}(K)$, the above theorem can be rephrased as follows.

Corollary 2. Let $K$ be any field, $n \geq 2$ an integer, and $f(x, y)=x y-y x$. Then $f\left(\mathbb{M}_{n}(K)\right)=$ $\left[\mathbb{M}_{n}(K), \mathbb{M}_{n}(K)\right]$.

It is therefore natural to ask what other polynomials $f$ have the property $f\left(\mathbb{M}_{n}(K)\right)=$ $\left[\mathbb{M}_{n}(K), \mathbb{M}_{n}(K)\right]$, or more generally, $f\left(\mathbb{M}_{n}(K)\right) \supseteq\left[\mathbb{M}_{n}(K), \mathbb{M}_{n}(K)\right]$. In this note we conjecture that this is the case for all nonzero multilinear polynomials $f$ (i.e., polynomials that are linear in each variable), provided that the degree of $f$ is at most $n+1$. We then prove this conjecture for multilinear polynomials of degree at most 3 , over fields with at least $n$ elements, and characterize the polynomials that satisfy $f\left(\mathbb{M}_{n}(K)\right)=\left[\mathbb{M}_{n}(K), \mathbb{M}_{n}(K)\right]$ in 
this situation. We also prove that for any unital ring $R$, the set of values of the polynomial $x z y-x y z+y z x-z y x$ on $\mathbb{M}_{n}(R)$ contains all matrices with the property that the sums of the elements along the diagonals above (and including) the main diagonal are 0.

Since $\left[\mathbb{M}_{n}(K), \mathbb{M}_{n}(K)\right]$ is a $K$-subspace of $\mathbb{M}_{n}(K)$, our conjecture and result above can be viewed as a partial answer to a more specific version of the following question of Lvov [4, Entry 1.98], which also has been attributed to Kaplansky (see [6]).

Question 3 (Lvov). Let $f$ be a multilinear polynomial over a field $K$. Is the set of values of $f$ on the matrix algebra $\mathbb{M}_{n}(K)$ a vector space?

Kanel-Belov, Malev, and Rowen [6] have answered this question in the case where $n=2$ and $K$ is quadratically closed (that is, every non-constant quadratic polynomial over $K$ has a root in $K)$. More specifically, they showed that in this case, the image of a multilinear polynomial must be one of $0, K \cdot I_{2}$ (where $I_{2}$ is the identity matrix), $\left[\mathbb{M}_{2}(K), \mathbb{M}_{2}(K)\right]$, or $\mathbb{M}_{2}(K)$.

Other special cases of Question 3 have been explored elsewhere. For instance, Khurana and Lam [8, Corollary 3.16] showed that the set of values of the multilinear polynomial $x y z-z y x$ on $\mathbb{M}_{n}(K)$, over an arbitrary field $K$, is all of $\mathbb{M}_{n}(K)$, when $n \geq 2$ (along with more general versions of this result for other rings $R$ in place of the field $K)$. Also, Kaplansky [7, Problem 16] asked whether there exists a nonzero multilinear polynomial over a field $K$ which always takes values in the center of $\mathbb{M}_{n}(K)$, or, equivalently, whether there is such a polynomial whose set of values on $\mathbb{M}_{n}(K)$ is the center of $\mathbb{M}_{n}(K)$. (This question arose in the study of polynomial identities on rings.) Such polynomials have indeed been constructed by Formanek [5] and Razmyslov [10].

All the results and questions mentioned above are stated for multilinear (rather than arbitrary) polynomials, since in general, the set of values of a polynomial on $\mathbb{M}_{n}(K)$ is not a subspace. More specifically, Chuang [3] showed that if $K$ is finite, then every subset $S$ of $\mathbb{M}_{n}(K)$ that contains 0 and is closed under conjugation is the image of some polynomial. Of course, such subsets $S$ of $\mathbb{M}_{n}(K)$ are generally not $K$-subspaces. Let us also give an example of a (non-multilinear) polynomial $f$ and an infinite field $K$ such that $f\left(\mathbb{M}_{n}(K)\right)$ is not a $K$-subspace of $\mathbb{M}_{n}(K)$.

Example 4. Let $K$ be an algebraically closed field, $n \geq 2$, and $f(x)=x^{n}$. For all distinct $1 \leq i, j \leq n$ we have $f\left(E_{i i}\right)=E_{i i}$ and $f\left(E_{i i}+E_{i j}\right)=E_{i i}+E_{i j}$, where $E_{i j}$ are the matrix units. Hence the $K$-subspace generated by $f\left(\mathbb{M}_{n}(K)\right)$ contains all $E_{i j}$, and therefore must be $\mathbb{M}_{n}(K)$. Thus, to conclude that $f\left(\mathbb{M}_{n}(K)\right)$ is not a subspace, it suffices to show that $f\left(\mathbb{M}_{n}(K)\right) \neq \mathbb{M}_{n}(K)$. Let $A \in \mathbb{M}_{n}(K)$ be a nonzero nilpotent matrix. Since 0 is the only eigenvalue of $A$, the Jordan canonical form of $A$ must be strictly upper-triangular, from which it follows that $A^{n}=0$. Thus, if $A=f(B)=B^{n}$ for some $B \in \mathbb{M}_{n}(K)$, then $B$ must be nilpotent, and hence $f(B)=B^{n}=0$ (by the above argument), contradicting $A \neq 0$. Therefore, $f\left(\mathbb{M}_{n}(K)\right)$ contains no nonzero nilpotent matrices, and hence $f\left(\mathbb{M}_{n}(K)\right)$ is not a subspace of $\mathbb{M}_{n}(K)$.

We conclude the Introduction by mentioning that there has been interest in generalizing Theorem 1 in other directions, particularly, in determining whether the same statement holds over an arbitrary (unital) ring. That is, given a ring $R$, can every matrix $M \in \mathbb{M}_{n}(R)$ 
having trace 0 be expressed as a commutator $M=A B-B A$, for some $A, B \in \mathbb{M}_{n}(R)$ ? Intriguingly, this question remained open until 2000, when Rosset and Rosset [11] produced a ring $R$ and a matrix $M \in \mathbb{M}_{2}(R)$ having trace 0 that cannot be expressed as a commutator. On the other hand, it turns out that over any unital ring $R$ and for any $n \geq 2$, every matrix $M \in \mathbb{M}_{n}(R)$ having trace 0 can be expressed as a sum of two commutators (see [9, Theorem 15]). To the best of our knowledge, however, there is still no classification of the rings $R$ with the property that every matrix with trace 0 is a commutator in $\mathbb{M}_{n}(R)$, despite continued attention to the question.

\section{Preliminaries}

Let us next define multilinear polynomials more rigorously, and then collect some basic facts about them.

Definition 5. Given a field $K$ and a positive integer $m$, we denote by $K\left\langle x_{1}, \ldots, x_{m}\right\rangle$ the $K$-algebra freely generated by the (non-commuting) variables $x_{1}, \ldots, x_{m}$. A polynomial $f\left(x_{1}, \ldots, x_{m}\right) \in K\left\langle x_{1}, \ldots, x_{m}\right\rangle$ is said to be multilinear (of degree $m$ ) if it is of the form

$$
f\left(x_{1}, \ldots, x_{m}\right)=\sum_{\sigma \in S_{m}} a_{\sigma} x_{\sigma(1)} x_{\sigma(2)} \ldots x_{\sigma(m)},
$$

where $S_{m}$ is the group of all permutations of $\{1, \ldots, m\}$ and $a_{\sigma} \in K$.

Some authors call these polynomials homogeneous multilinear. The motivation for calling such polynomials "multilinear" is that given any $K$-algebra $A$, any element $f\left(x_{1}, \ldots, x_{m}\right) \in$ $K\left\langle x_{1}, \ldots, x_{m}\right\rangle$ can be viewed as a map $f: A \times \cdots \times A \rightarrow A$ by evaluating the $x_{i}$ on elements of $A$, and it is easy to see that the polynomials of the above form are precisely the ones that give rise to maps $f: A \times \cdots \times A \rightarrow A$ that are linear in each variable (for every $K$-algebra $A$ ). That is, maps $f$ such that for all $a, b \in K, j \in\{1, \ldots, m\}$, and $r_{1}, \ldots, r_{m}, r_{j}^{\prime} \in A$, we have

$$
\begin{gathered}
f\left(r_{1}, \ldots, r_{j-1}, a r_{j}+b r_{j}^{\prime}, r_{j+1}, \ldots, r_{m}\right) \\
=a f\left(r_{1}, \ldots, r_{j-1}, r_{j}, r_{j+1}, \ldots, r_{m}\right)+b f\left(r_{1}, \ldots, r_{j-1}, r_{j}^{\prime}, r_{j+1}, \ldots, r_{m}\right) .
\end{gathered}
$$

Given a polynomial $f\left(x_{1}, \ldots, x_{m}\right) \in K\left\langle x_{1}, \ldots, x_{m}\right\rangle$ and $K$-algebra $A$, we set $f(A)=$ $\left\{f\left(r_{1}, \ldots, r_{m}\right) \mid r_{1}, \ldots, r_{m} \in A\right\}$. Also, for any associative $\operatorname{ring} R$ we shall denote by $[r, p]$ the commutator $r p-p r$ of $r, p \in R$, denote by $[R, R]$ the additive subgroup of $R$ generated by the commutators, and let $[r, R]=\{[r, p] \mid p \in R\}$ for $r \in R$.

In the next lemma we record a couple basic facts about multilinear polynomials for later reference. Both claims follow immediately from Definition 5 .

Lemma 6. Let $K$ be a field, $A$ a $K$-algebra, $m$ a positive integer, and $f\left(x_{1}, \ldots, x_{m}\right) \in$ $K\left\langle x_{1}, \ldots, x_{m}\right\rangle$ a multilinear polynomial. Also, let $r_{1}, \ldots, r_{m} \in A$ be arbitrary elements. Then the following hold.

(1) For any $a \in K$ we have af $\left(r_{1}, \ldots, r_{m}\right)=f\left(a r_{1}, \ldots, r_{m}\right)$.

(2) For any invertible $p \in A$ we have $p f\left(r_{1}, \ldots, r_{m}\right) p^{-1}=f\left(p r_{1} p^{-1}, \ldots, p r_{m} p^{-1}\right)$. 
The next lemma, which is an easy consequence of Theorem 1, describes the sets of values of multilinear polynomials of degree at most 2 on matrix algebras.

Lemma 7. Let $K$ be a field, $m$ a positive integer, and $f\left(x_{1}, \ldots, x_{m}\right) \in K\left\langle x_{1}, \ldots, x_{m}\right\rangle a$ multilinear polynomial.

(1) If $m=1$, then $f\left(\mathbb{M}_{n}(K)\right) \in\left\{0, \mathbb{M}_{n}(K)\right\}$.

(2) If $m=2$, then $f\left(\mathbb{M}_{n}(K)\right) \in\left\{0,\left[\mathbb{M}_{n}(K), \mathbb{M}_{n}(K)\right], \mathbb{M}_{n}(K)\right\}$.

Proof. If $m=1$, then $f$ must be of the form $f(x)=a x$ for some $a \in K$, from which (1) follows.

If $m=2$, then $f$ must be of the form $f(x, y)=a x y+b y x$ for some $a, b \in K$. If $a+b \neq 0$, then $f\left(I_{n}, Y\right)=(a+b) Y$ for all $Y \in \mathbb{M}_{n}(K)$, which implies that $f\left(\mathbb{M}_{n}(K)\right)=\mathbb{M}_{n}(K)$. While, if $a+b=0$, then $f(x, y)=a(x y-y x)$, which has the same image as the polynomial $x y-y x$, as long as $a \neq 0$, by Lemma 6(1). Statement (2) now follows from Corollary 2.

The following fact will be useful in subsequent arguments. For a polynomial $f\left(x_{1}, \ldots, x_{m}\right) \in$ $K\left\langle x_{1}, \ldots, x_{m}\right\rangle$ and $1 \leq l \leq m$ we view $f\left(x_{1}, \ldots, x_{l}, 1, \ldots, 1\right)$ as a polynomial in $K\left\langle x_{1}, \ldots, x_{l}\right\rangle$.

Corollary 8. Let $K$ be a field, $m$ a positive integer, and

$$
f\left(x_{1}, \ldots, x_{m}\right)=\sum_{\sigma \in S_{m}} a_{\sigma} x_{\sigma(1)} x_{\sigma(2)} \ldots x_{\sigma(m)} \in K\left\langle x_{1}, \ldots, x_{m}\right\rangle
$$

a multilinear polynomial.

(1) If $\sum_{\sigma \in S_{m}} a_{\sigma} \neq 0$, then $f\left(\mathbb{M}_{n}(K)\right)=\mathbb{M}_{n}(K)$.

(2) If $m \geq 2$ and $f\left(x_{1}, x_{2}, 1, \ldots, 1\right) \neq 0$, then $f\left(\mathbb{M}_{n}(K)\right) \in\left\{\left[\mathbb{M}_{n}(K), \mathbb{M}_{n}(K)\right], \mathbb{M}_{n}(K)\right\}$.

Proof. This follows from Lemma 7 upon noting that $f\left(x_{1}, 1, \ldots, 1\right)=\left(\sum_{\sigma \in S_{m}} a_{\sigma}\right) x_{1}$, and $f\left(x_{1}, x_{2}, 1, \ldots, 1\right)=b x_{1} x_{2}+c x_{2} x_{1}$, for some $b, c \in K$.

\section{A conjecture}

The main goal of this section is to justify a conjecture regarding the set of values of certain multilinear polynomials. We shall first require the following result of Amitsur and Rowen [2, Proposition 1.8] to prove a fact about the linear spans of the images of our polynomials.

Proposition 9 (Amitsur/Rowen). Let $D$ be a division ring, $n \geq 2$ an integer, and $A \in$ $\mathbb{M}_{n}(D)$. Then $A$ is similar to a matrix in $\mathbb{M}_{n}(D)$ with at most one nonzero entry on the main diagonal. In particular, if $A$ has trace zero, then it is similar to a matrix in $\mathbb{M}_{n}(D)$ with only zeros on the main diagonal.

Proposition 10. Let $K$ be a field, $n \geq 2$ and $m \geq 1$ integers, and $f\left(x_{1}, \ldots, x_{m}\right)$ a nonzero multilinear polynomial in $K\left\langle x_{1}, \ldots, x_{m}\right\rangle$. If $n \geq m-1$, then the $K$-subspace $\left\langle f\left(\mathbb{M}_{n}(K)\right)\right\rangle$ of $\mathbb{M}_{n}(K)$ generated by $f\left(\mathbb{M}_{n}(K)\right)$ contains $\left[\mathbb{M}_{n}(K), \mathbb{M}_{n}(K)\right]$. 
Proof. Write

$$
f\left(x_{1}, \ldots, x_{m}\right)=\sum_{\sigma \in S_{m}} a_{\sigma} x_{\sigma(1)} x_{\sigma(2)} \ldots x_{\sigma(m)},
$$

for some $a_{\sigma} \in K$. Since $f$ is nonzero, upon relabeling the variables if necessary, we may assume that $a_{1} \neq 0$. Furthermore, by Lemma 7 , if $m \leq 2$, then $\left[\mathbb{M}_{n}(K), \mathbb{M}_{n}(K)\right] \subseteq f\left(\mathbb{M}_{n}(K)\right)$ for any $n \geq 2$. We therefore may assume that $m \geq 3$.

Let $i, j \in\{1, \ldots, n\}$ be distinct. Since $n \geq m-1 \geq 2$, we can find distinct elements $l_{1}, \ldots, l_{m-3} \in\{1, \ldots, n\} \backslash\{i, j\}$. Letting $E_{k l}$ denote the matrix units, we then have

$$
\begin{gathered}
f\left(E_{i i}, E_{i j}, E_{j l_{1}}, E_{l_{1} l_{2}}, \ldots, E_{l_{m-4} l_{m-3}}, E_{l_{m-3} j}\right) \\
=a_{1} E_{i i} E_{i j} E_{j l_{1}} E_{l_{1} l_{2}} \ldots E_{l_{m-4} l_{m-3}} E_{l_{m-3} j}+0=a_{1} E_{i j},
\end{gathered}
$$

since multiplying $E_{i i}, E_{i j}, E_{j l_{1}}, E_{l_{1} l_{2}}, \ldots, E_{l_{m-4} l_{m-3}}, E_{l_{m-3} j}$ in any other order yields 0 . (If $m=3$, then we interpret the above equation as $f\left(E_{i i}, E_{i j}, E_{j j}\right)=a_{1} E_{i j}$. $)$ Therefore, $E_{i j} \in$ $f\left(\mathbb{M}_{n}(K)\right)$ for all distinct $i$ and $j$, and hence $\left\langle f\left(\mathbb{M}_{n}(K)\right)\right\rangle$ contains all matrices with zeros on the main diagonal. Now, Lemma 6 implies that $\left\langle f\left(\mathbb{M}_{n}(K)\right)\right\rangle$ is closed under conjugation. Hence, by Proposition 9, we have $\left[\mathbb{M}_{n}(K), \mathbb{M}_{n}(K)\right] \subseteq\left\langle f\left(\mathbb{M}_{n}(K)\right)\right\rangle$.

The claim in the above proposition does not in general hold when $n<m-1$. For example, it is known that the set of values of the polynomial $(x y-y x)^{2}$ on $\mathbb{M}_{2}(K)$ is contained in the center $K \cdot I_{2}$ (see [7]). The same is true of the linearization

$$
f\left(x_{1}, x_{2}, y_{1}, y_{2}\right)=\left[x_{1}, y_{1}\right]\left[x_{2}, y_{2}\right]+\left[x_{1}, y_{2}\right]\left[x_{2}, y_{1}\right]+\left[x_{2}, y_{1}\right]\left[x_{1}, y_{2}\right]+\left[x_{2}, y_{2}\right]\left[x_{1}, y_{1}\right]
$$

of this polynomial. Thus, $\left\langle f\left(\mathbb{M}_{2}(K)\right)\right\rangle=K \cdot I_{2} \nsupseteq\left[\mathbb{M}_{2}(K), \mathbb{M}_{2}(K)\right]$.

An affirmative answer to Question 3 would imply that in the above proposition, if $n \geq$ $m-1$, then $f\left(\mathbb{M}_{n}(K)\right)$, and not just $\left\langle f\left(\mathbb{M}_{n}(K)\right)\right\rangle$, contains $\left[\mathbb{M}_{n}(K), \mathbb{M}_{n}(K)\right]$. Since it is suspected that the question does have an affirmative answer (e.g., see [6]), we make the following conjecture.

Conjecture 11. Let $K$ be a field, $n \geq 2$ and $m \geq 1$ integers, and $f\left(x_{1}, \ldots, x_{m}\right)$ a nonzero multilinear polynomial in $K\left\langle x_{1}, \ldots, x_{m}\right\rangle$. If $n \geq m-1$, then $f\left(\mathbb{M}_{n}(K)\right) \supseteq\left[\mathbb{M}_{n}(K), \mathbb{M}_{n}(K)\right]$.

Lemma 7 shows that this conjecture holds for $m<3$. In the next section we shall show that it holds for $m=3$ as well, if $K$ has at least $n$ elements.

\section{The three-variable case}

We shall require another fact proved by Amitsur and Rowen [2, Lemma 1.2].

Lemma 12 (Amitsur/Rowen). Let $K$ be a field and $n \geq 2$ an integer. Suppose that $A=$ $\left(a_{i j}\right) \in \mathbb{M}_{n}(K)$ is a diagonal matrix with $a_{i i} \neq a_{j j}$ for $i \neq j$. Then $\left[A, \mathbb{M}_{n}(K)\right]$ consists of all the matrices with only zeros on the main diagonal.

We are now ready for our main result. 
Theorem 13. Let $n \geq 2$ be an integer, $K$ a field with at least $n$ elements, and $f \in K\langle x, y, z\rangle$ any nonzero multilinear polynomial. Then $f\left(\mathbb{M}_{n}(K)\right)$ contains every matrix having trace 0 .

Proof. If $f$ has degree at most 2, then this follows from Lemma 7 , Thus, let us assume that the degree of $f$ is 3 . We can then write

$$
f(x, y, z)=a x y z+b x z y+c y x z+d y z x+e z x y+g z y x(a, b, c, d, e, g \in K) .
$$

If $a+b+c+d+e+g \neq 0$, then $\left[\mathbb{M}_{n}(K), \mathbb{M}_{n}(K)\right] \subseteq f\left(\mathbb{M}_{n}(K)\right)$, Corollary $8(1)$. Let us therefore suppose that $a+b+c+d+e+g=0$. In this case

$$
f(x, y, z)=a(x y z-z y x)+b(x z y-z y x)+c(y x z-z y x)+d(y z x-z y x)+e(z x y-z y x) .
$$

Moreover, if any of $f(1, y, z), f(x, 1, z)$, or $f(x, y, 1)$ are nonzero, then by Corollary $8(2)$, $\left[\mathbb{M}_{n}(K), \mathbb{M}_{n}(K)\right] \subseteq f\left(\mathbb{M}_{n}(K)\right)$. Thus, let us assume that

$$
0=f(1, y, z)=a(y z-z y)+c(y z-z y)+d(y z-z y)=(a+c+d)(y z-z y),
$$

which implies that $0=a+c+d$. Setting $0=f(x, 1, z)$ and $0=f(x, y, 1)$ we similarly get $0=a+b+c$ and $0=a+b+e$. Solving the resulting system of equations gives $b=d, c=e$, and $a=-b-c$. Therefore,

$$
\begin{gathered}
f(x, y, z)=(-b-c)(x y z-z y x)+b(x z y-z y x+y z x-z y x)+c(y x z-z y x+z x y-z y x) \\
=b(x z y-z y x+y z x-x y z)+c(y x z-z y x+z x y-x y z) \\
=b(x[z, y]-[z, y] x)+c(z[x, y]-[x, y] z)=b[x,[z, y]]+c[z,[x, y]] .
\end{gathered}
$$

Now, since $f(x, y, z) \neq 0$, renaming the variables, if necessary, we may assume that $b \neq 0$. Then, by Lemma $6(1), f(x, y, z)$ and $b^{-1} f(x, y, z)$ have the same set of values. Therefore, we may assume that $f(x, y, z)=[x,[z, y]]+b[z,[x, y]]$ for some $b \in K$.

Let $A \in \mathbb{M}_{n}(K)$ be a matrix having trace 0 . We wish to show that $A \in f\left(\mathbb{M}_{n}(K)\right)$. By Proposition 9, $A$ is conjugate to a matrix $A^{\prime} \in \mathbb{M}_{n}(K)$ with only zeros on the main diagonal. Hence, by Lemma 6(2), to conclude the proof it is enough to show that that $A^{\prime} \in f\left(\mathbb{M}_{n}(K)\right)$. By our assumption on $K$, we can find a diagonal matrix $M \in \mathbb{M}_{n}(K)$ with distinct elements of $K$ on its main diagonal. By Lemma 12, there is some $B \in \mathbb{M}_{n}(K)$ such that $A^{\prime}=[M, B]$. Now, write $B=C+D$, where $C$ has only zeros on the main diagonal and $D$ is diagonal. Then

$$
A^{\prime}=[M, B]=[M, C+D]=[M, C]+[M, D]=[M, C],
$$

since $M$ commutes with all diagonal matrices. Using Lemma 12 once again, we can find a matrix $E \in \mathbb{M}_{n}(K)$ such that $C=[E, M]$. Finally, we have

$$
f(M, M, E)=[M,[E, M]]+b[E,[M, M]]=[M,[E, M]]=[M, C]=A^{\prime},
$$

as desired.

Let us next describe the degree-three multilinear polynomials $f$ satisfying $f\left(\mathbb{M}_{n}(K)\right)=$ $\left[\mathbb{M}_{n}(K), \mathbb{M}_{n}(K)\right]$. 
Lemma 14. Let $n \geq 2$ be an integer, $K$ a field, and

$$
f\left(x_{1}, x_{2}, x_{3}\right)=\sum_{\sigma \in S_{3}} a_{\sigma} x_{\sigma(1)} x_{\sigma(2)} x_{\sigma(3)} \in K\left\langle x_{1}, x_{2}, x_{3}\right\rangle
$$

a multilinear polynomial of degree 3. Then $f\left(\mathbb{M}_{n}(K)\right) \subseteq\left[\mathbb{M}_{n}(K), \mathbb{M}_{n}(K)\right]$ if and only if $\sum_{\sigma \in S_{3}} a_{\sigma}=0=\sum_{\sigma \in A_{3}} a_{\sigma}$, where $A_{3} \subseteq S_{3}$ is the alternating subgroup.

Proof. By Corollary $8(1)$, we may assume that $\sum_{\sigma \in S_{3}} a_{\sigma}=0$. Now, suppose that $\sum_{\sigma \in A_{3}} a_{\sigma}=$ 0 . Then we must also have $\sum_{\sigma \in S_{3} \backslash A_{3}} a_{\sigma}=\sum_{\sigma \in S_{3}} a_{\sigma}-\sum_{\sigma \in A_{3}} a_{\sigma}=0$. Therefore,

$$
\begin{gathered}
f\left(x_{1}, x_{2}, x_{3}\right)=\sum_{\sigma \in A_{3}} a_{\sigma} x_{\sigma(1)} x_{\sigma(2)} x_{\sigma(3)}+\sum_{\sigma \in S_{3} \backslash A_{3}} a_{\sigma} x_{\sigma(1)} x_{\sigma(2)} x_{\sigma(3)} \\
=\sum_{\sigma \in A_{3} \backslash\{1\}} a_{\sigma}\left(x_{\sigma(1)} x_{\sigma(2)} x_{\sigma(3)}-x_{1} x_{2} x_{3}\right)+\sum_{\sigma \in S_{3} \backslash\left(A_{3} \cup\{(12)\}\right)} a_{\sigma}\left(x_{\sigma(1)} x_{\sigma(2)} x_{\sigma(3)}-x_{2} x_{1} x_{3}\right) \\
=a_{(123)}\left(x_{2} x_{3} x_{1}-x_{1} x_{2} x_{3}\right)+a_{(132)}\left(x_{3} x_{1} x_{2}-x_{1} x_{2} x_{3}\right) \\
+a_{(13)}\left(x_{3} x_{2} x_{1}-x_{2} x_{1} x_{3}\right)+a_{(23)}\left(x_{1} x_{3} x_{2}-x_{2} x_{1} x_{3}\right) \\
=a_{(123)}\left[x_{2} x_{3}, x_{1}\right]+a_{(132)}\left[x_{3}, x_{1} x_{2}\right]+a_{(13)}\left[x_{3}, x_{2} x_{1}\right]+a_{(23)}\left[x_{1} x_{3}, x_{2}\right] .
\end{gathered}
$$

Thus, $f\left(\mathbb{M}_{n}(K)\right) \subseteq\left[\mathbb{M}_{n}(K), \mathbb{M}_{n}(K)\right]$.

On the other hand, $f\left(E_{11}, E_{12}, E_{21}\right)=a_{1} E_{11}+a_{(123)} E_{11}+a_{(132)} E_{22}$ has trace $\sum_{\sigma \in A_{3}} a_{\sigma}$. Hence, if this sum is not zero, then $f\left(\mathbb{M}_{n}(K)\right) \nsubseteq\left[\mathbb{M}_{n}(K), \mathbb{M}_{n}(K)\right]$.

Applying Theorem 13 to this lemma we obtain the following.

Corollary 15. Let $n \geq 2$ be an integer, $K$ a field with at least $n$ elements, and

$$
f\left(x_{1}, x_{2}, x_{3}\right)=\sum_{\sigma \in S_{3}} a_{\sigma} x_{\sigma(1)} x_{\sigma(2)} x_{\sigma(3)} \in K\left\langle x_{1}, x_{2}, x_{3}\right\rangle
$$

a nonzero multilinear polynomial of degree 3 . Then $f\left(\mathbb{M}_{n}(K)\right)=\left[\mathbb{M}_{n}(K), \mathbb{M}_{n}(K)\right]$ if and only if $\sum_{\sigma \in S_{3}} a_{\sigma}=0=\sum_{\sigma \in A_{3}} a_{\sigma}$, where $A_{3} \subseteq S_{3}$ is the alternating subgroup.

We note that in general the condition $\sum_{\sigma \in S_{m}} a_{\sigma}=0=\sum_{\sigma \in A_{m}} a_{\sigma}$ does not characterize the nonzero multilinear polynomials $f\left(x_{1}, \ldots, x_{m}\right)$ satisfying $f\left(\mathbb{M}_{n}(K)\right)=\left[\mathbb{M}_{n}(K), \mathbb{M}_{n}(K)\right]$. For example, if $m=2$, then $\sum_{\sigma \in S_{m}} a_{\sigma}=0=\sum_{\sigma \in A_{m}} a_{\sigma}$ implies that $f\left(x_{1}, x_{2}\right)=0$. Also, $f\left(x_{1}, x_{2}, x_{3}, x_{4}\right)=x_{1} x_{2} x_{3} x_{4}-x_{4} x_{3} x_{2} x_{1}$ satisfies $\sum_{\sigma \in S_{4}} a_{\sigma}=0=\sum_{\sigma \in A_{4}} a_{\sigma}$, but $f\left(\mathbb{M}_{n}(K)\right) \nsubseteq$ $\left[\mathbb{M}_{n}(K), \mathbb{M}_{n}(K)\right]$ for any $n \geq 2$, since $f\left(E_{11}, E_{12}, E_{22}, E_{21}\right)=E_{11} \notin\left[\mathbb{M}_{n}(K), \mathbb{M}_{n}(K)\right]$.

Let us conclude with a fact about the image of the degree-three multilinear polynomial $[x,[y, z]]$ on matrices over an arbitrary ring. We first require the following lemma.

Lemma 16. Let $R$ be a unital ring, let $n \geq 2$ be an integer, and let $A=\left(a_{i j}\right) \in \mathbb{M}_{n}(R)$ be such that for each $j \in\{0,1, \ldots, n-1\}$ we have $\sum_{i=1}^{n-j} a_{i, i+j}=0$ (i.e., sums of the elements along the diagonals above and including the main diagonal are 0$)$. Then $A=D X-X D$ for some $D \in \mathbb{M}_{n}(R)$ and $X=\sum_{i=1}^{n-1} E_{i+1, i}$, where $E_{i j}$ are the matrix units. 
Proof. Letting $Z=\sum_{i=1}^{n-1} E_{i, i+1}$ we have $Z X=\sum_{i=1}^{n-1} E_{i i}=I-E_{n n}$. For any $l \in\{0,1, \ldots, n-$ $1\}$ and $k \in\{1,2, \ldots, n\}$ we then have

$$
E_{k k} X^{l} A Z^{l} E_{n n}=E_{k k}\left(\sum_{i=1}^{n-l} E_{i+l, i}\right) A\left(\sum_{i=1}^{n-l} E_{i, i+l}\right) E_{n n}=E_{k, k-l} A E_{n-l, n}=a_{k-l, n-l} E_{k n}
$$

if $l<k$, and $E_{k k} X^{l} A Z^{l} E_{n n}=0 \cdot A Z^{l} E_{n n}=0$ if $l \geq k$.

Letting $D=\sum_{i=0}^{n-2} X^{i} A Z^{i+1}$, we have

$$
\begin{gathered}
D X-X D=\left(\sum_{i=0}^{n-2} X^{i} A Z^{i}\right) Z X-\sum_{i=0}^{n-2} X^{i+1} A Z^{i+1} \\
=\left(A+\sum_{i=1}^{n-2} X^{i} A Z^{i}\right)\left(I-E_{n n}\right)-\sum_{i=1}^{n-1} X^{i} A Z^{i}=A-\left(\sum_{i=0}^{n-2} X^{i} A Z^{i}\right) E_{n n}-X^{n-1} A Z^{n-1} \\
=A-\left(\sum_{i=0}^{n-2} X^{i} A Z^{i}\right) E_{n n}-X^{n-1} A E_{1 n}=A-\left(\sum_{i=0}^{n-1} X^{i} A Z^{i}\right) E_{n n} .
\end{gathered}
$$

Now, for every $k \in\{1,2, \ldots, n\}$ we have

$$
E_{k k}\left(\sum_{i=0}^{n-1} X^{i} A Z^{i}\right) E_{n n}=\sum_{i=0}^{k-1} E_{k k} X^{i} A Z^{i} E_{n n}=\left(\sum_{i=0}^{k-1} a_{k-i, n-i}\right) E_{k n}=\left(\sum_{i=1}^{k} a_{i, i+(n-k)}\right) E_{k n},
$$

by the computation in the first paragraph. Finally, the last sum is 0 , by hypothesis on $A$, and hence $\left(\sum_{i=0}^{n-1} X^{i} A Z^{i}\right) E_{n n}=0$, showing that $D X-X D=A$.

Theorem 17. Let $R$ be unital ring, let $n \geq 2$ be an integer, let $f(x, y, z)=[x,[z, y]]$, and let $A=\left(a_{i j}\right) \in \mathbb{M}_{n}(R)$ be such that for each $j \in\{0,1, \ldots, n-1\}$ we have $\sum_{i=1}^{n-j} a_{i, i+j}=0$. Then $A=f(D, X, Y)$ for some $D \in \mathbb{M}_{n}(R), X=\sum_{i=1}^{n-1} E_{i+1, i}$, and $Y=\sum_{i=1}^{n}(i-1) E_{i i}$.

Proof. For any matrix $M=\left(m_{i j}\right) \in \mathbb{M}_{n}(K)$ we have

$$
\begin{gathered}
{[Y, M]=\left(\begin{array}{ccccc}
0 & 0 & 0 & \ldots & 0 \\
m_{21} & m_{22} & m_{23} & \ldots & m_{2 n} \\
2 m_{31} & 2 m_{32} & 2 m_{33} & \ldots & 2 m_{3 n} \\
\vdots & \vdots & \vdots & \ddots & \vdots
\end{array}\right)-\left(\begin{array}{ccccc}
0 & m_{12} & 2 m_{13} & \ldots & (n-1) m_{1 n} \\
0 & m_{22} & 2 m_{23} & \ldots & (n-1) m_{2 n} \\
0 & m_{32} & 2 m_{33} & \ldots & (n-1) m_{3 n} \\
\vdots & \vdots & \vdots & \ddots & \vdots
\end{array}\right)} \\
=\left((i-1) m_{i j}\right)-\left((j-1) m_{i j}\right)=\left((i-j) m_{i j}\right) .
\end{gathered}
$$

Hence, in particular, $[Y, X]=X$.

Now, by Lemma [16, $A=[D, X]$ for some $D \in \mathbb{M}_{n}(R)$. We therefore have

$$
f(D, X, Y)=[D,[Y, X]]=[D, X]=A,
$$

proving the statement.

This argument extends easily to all polynomials of the form $\left[x_{1},\left[x_{2}, \ldots,\left[x_{m-1}, x_{m}\right]\right] \ldots\right]$. 


\section{Acknowledgements}

I am grateful to Mikhail Chebotar for bringing Question 3 to my attention, and to the referee for suggesting a better way to prove the main result.

\section{References}

[1] A. A. Albert and B. Muckenhoupt, On matrices of trace zero, Michigan Math. J. 4 (1957) 1-3.

[2] S. A. Amitsur and L. H. Rowen, Elements of reduced trace 0, Israel J. Math. 87 (1994) $161-179$.

[3] C.-L. Chuang, On ranges of polynomials in finite matrix rings, Proc. Amer. Math. Soc. 110 (1990) 293-302.

[4] The Dniester Notebook: Unsolved Problems in the Theory of Rings and Modules, Mathematics Institute, Russian Academy of Sciences Siberian Branch, Novosibirsk, Fourth Edition, 1993.

[5] E. Formanek, Central polynomials for matrix rings, J. Algebra 23 (1972) 129-132.

[6] A. Kanel-Belov, S. Malev, and L. Rowen, The images of non-commutative polynomials evaluated on $2 \times 2$ matrices, Proc. Amer. Math. Soc. 140 (2012) 465-478.

[7] I. Kaplansky, "Problems in the Theory of Rings" Revisited, Amer. Math. Monthly 77 (1970) 445-454.

[8] D. Khurana and T. Y. Lam, Generalized commutators in matrix rings, Lin. Multilin. Alg. 60 (2012) 797-827.

[9] Z. Mesyan, Commutator rings, Bull. Austral. Math Soc. 74 (2006) 279-288.

[10] Y. Razmyslov, On a problem of Kaplansky, Izv. Akad. Nauk SSSR., Ser. Mat. 37 (1973) $483-501$.

[11] M. Rosset and S. Rosset, Elements of trace zero that are not commutators, Comm. Algebra 28 (2000) 3059-3072.

[12] K. Shoda, Einige Sätze über Matrizen, Japan J. Math 13 (1936) 361-365.

Department of Mathematics

University of Colorado

Colorado Springs, CO 80918

USA

Email: zmesyan@uccs.edu 